\section{Psoriasispatienten in Diabetesgefahr}

Kalifornische Forscher vermuten eine Verbindung zwischen Psoriasis und Typ-2Diabetes. Die Analyse von 27 Diabetes-Studien zeigte erhöhte Prävalenzen, von 53\% bei milder Psoriasis bis $97 \%$ bei schweren Verlaufsformen. Die systemische Entzündung erhöht womöglich die Spiegel bestimmter Interleukine und des Tumor-Nekrose-Faktors (TNF), was sich negativ auf den Glukosestoffwechsel auswirkt. Zudem könnten veränderte Signalwege der Th1Helferzellen und dysregulierte oxidative Mechanismen die Glukosetoleranz stören.

Armstrong AW et al, Arch Dermatol 2012

\section{Wunden heilen oben ohne genauso gut}

Mit oder ohne Verband, spielt für das Infektionsrisiko keine Rolle. Der Vergleich von 2594 Wunden aller Kontaminations- und Schweregrade, und mit Materialien vom Gazeverband über Alginat oder Hydrokolloiden bis zu antimikrobiellen und Matrixauflagen, zeigte: Kein Verband war einem anderen überlegen. Selbst an der Luft belassene Wunden waren nicht öfter infiziert. Lediglich die Absorptionsfähigkeit und die Kosten machen den Unterschied.

Walter CJ et al, Br J Surg 2012, 99: 1185-94

\section{Schmerzmittel: Missbrauch im Sport!}

Um die Karriere erfolgreich zu bestreiten, müssen Sportler immer häufiger oberhalb der Schmerzgrenze agieren: 60\% aller Fußballer griffen während der letzten WM prophylaktisch zu Analgetika, so die FIFA. Bereits $20-25 \%$ der unter 17-Jährigen greifen während offiziellen Turnieren regelmäßig zur Schmerzpille, erstaunlicherweise Frauen mit 30,7\% noch häufiger. Klar vorne in der Rangliste stehen NSAR wie Diclofenac, selektive COX-2-Hemmer/Nimesulid oder ASS.

Deutscher Schmerzkongress 2012,

Mannheim

Belastungstest nach Endoprothetik

Wie viel Sport verträgt

die neue Hüfte?

(0) Getty Images/iStockphoto/thinkstock.com

Zehn Jahre nach einer Hüft-Op. ist die Funktionsfähigkeit bei Personen, die gelenkbelastende Sportarten betreiben, besser als bei weniger Aktiven; allerdings auf Kosten der Haltbarkeit des künstlichen Gelenks.

Wissenschaftler der Universität Marseille beobachteten 210 Patienten (18-75 Jahre) zur Studie der Belastbarkeit nach der Endoprothetik über durchschnittlich elf Jahre. Alle Patienten erhielten eine zementfrei verankerte Prothese mit Keramikkopf und einem Titan-Acetabulum mit ultrahochmolekularem Polyethylen. 70 Patienten gingen postoperativ weiterhin ihren gelenkstrapazierenden Sportarten nach, u.a. Joggen, Fußball oder Skifahren. Ihre Daten verglichen die Forscher mit 140 Hüftoperierten mit geringem Aktivitätslevel.

Evaluiert wurde neben Funktionsfähigkeit (Harris Hip Score (HHS): $<70=$ schlecht; $\max .100=$ hervorragend) und Lebensqualität (Hip Osteoarthritis Outcome Score (HOOS): 0-100) auch die Ver- schleißrate und die Zeit bis zum mechanischen Versagen der Prothese.

Die Funktionalität hatte sich bei stärker beanspruchten Gelenken nach der Op. von 54 auf 88 HHS-Punkte gebessert, bei den gering Aktiven nur von 55 auf 69. Auch der HOOS fiel bei den Sportlicheren in drei von fünf Subskalen besser aus, und zwar hinsichtlich der Symptome, der Alltagsaktivitäten und der Beschwerden bei Sport- und Freizeitaktivitäten. Vergleichbare Werte ergaben sich bei Schmerz und Lebensqualität. Auch die Dislokationsrate war in beiden Gruppen ähnlich.

Allerdings zeigten sich bei den Aktiven, und insbesondere bei Männern, höhere Verschleißraten nach 10-15 Jahren, mit $1,62 \mathrm{~mm}$ gegenüber $0,74 \mathrm{~mm}$ Polyethylenabrieb bei den Unsportlicheren. Auch die Lebensdauer der Prothesen war durch die höhere Belastung verkürzt: Der Preis für gelenkbelastenden Sport war ein 3,6-faches Risiko für ein mechanisches Versagen. (st)

Ollivier M et al, Clin Orthop Relat Res 2012, 470:3060-3066

\title{
Bei adipösen Frauen
}

\section{Besserer Sex nach Magenverkleinerung}

Der Gewichtsverlust nach einer Magenverkleinerung beeinflusst den Zyklus nicht so stark wie bisher vermutet: Lediglich die follikuläre Phase verkürzt sich, und die Frauen haben mehr Spaß am Sex!

Stark adipösen Frauen, die Probleme haben, schwanger zu werden, wird empfohlen, ihr Gewicht zu reduzieren. Gynäkologen prüften nun in einer kleinen prospektiven Studie, ob sich durch eine Magenverkleinerung Reproduktionsparameter ändern. Zwei Jahre lang wurden 29 Frauen beobachtet, die im Mittel einen BMI von
$49 \mathrm{~kg} / \mathrm{m}^{2}(132 \mathrm{~kg})$ hatten. Das Ergebnis: Weder die Ovulationsfrequenz noch die Qualität der Lutealphase änderten sich durch den Eingriff. Die Zyklusdauer, die bei Adipösen meist verlängert ist, hatte sich zunächst signifikant verkürzt, was aber ein Jahr nach der Op. nicht mehr zutraf. Eine Veränderung erfuhr vor allem die Libido der Probandinnen: ihre Lust auf Sex stieg! Die Verbesserung um 28\% habe vermutlich dazu beigetragen, dass fünf Frauen im Verlauf der Studie schwanger wurden.

(pl) 